\title{
Introduction to EANM guideline for the preparation of an Investigational Medicinal Product Dossier (IMPD)
}

\author{
Arturo Chiti
}

Published online: 21 August 2014

(C) Springer-Verlag Berlin Heidelberg 2014

Radiopharmaceuticals are the core of nuclear medicine. This is a statement that no one would or could refute. In fact, during the evolution of our discipline, we came to realise at a certain point in time that "tracers" no longer exist. Although every nuclear medicine practitioner has knowledge of tracer principles, we had to abandon this term, move forward and begin to use "radiopharmaceuticals" for diagnostic and therapeutic purposes.

Over the years, we have also become aware that it is not only the definition of the substances which we use for diagnosis and treatment that has changed. There has in fact also been a great impact on our practice in terms of the set of rules and procedures that must be respected when using radiopharmaceuticals. We have had to use the same substances, with the same radiation protection rules, but with the addition of pharmaceutical rules. This change in behaviour is still ongoing. It has reached different levels of development in different countries, but the goal is always the same: to increase patient safety.

I am convinced that we shall benefit from this important change in our daily life, in research and in clinical practice, but we shall have to drive the process. Put another way, while it is good to alter the rules if doing so enables us to provide better health care, we have to be an integral part of, and contribute to, this process. It is not acceptable simply to follow rules that were designed for non-radioactive pharmaceuticals.

With this aim in mind, the European Society of Nuclear Medicine (EANM), first and foremost through the Radiopharmacy Committee but with the help of all other Committees, started to work very hard to make the European authorities aware that radiopharmaceuticals are not exactly pharmaceuticals. By means of various activities and publications, we have been able to influence some of the legislators' activities, but many efforts will still be required over the coming years.

The guideline for the preparation of an Investigational Medicinal Product Dossier (IMPD) is the result of one of these initiatives. Although not always an easy read for a physician, this document gives a clear and concise overview of the status of legislation on radiopharmaceuticals in Europe and, above all, provides immense help in preparing an IMPD. This step is critical when preparing the documents for clinical trials, and two precious practical examples of IMPDs are included as supplementary material.

Although not all the nuclear medicine departments in Europe will need to prepare an IMPD, this paper gives a clear idea of how to deal with the concepts of quality and risk assessment in our practice.

\footnotetext{
A. Chiti $(\bowtie)$

Nuclear Medicine, Humanitas Research Hospital, Via Manzoni 56, 20089 Rozzano, MI, Italy

e-mail: arturo.chiti@humanitas.it
} 